\title{
NOTAS CARIOLÓGICAS SOBRE ALGUNAS COMPUESTAS DE MARRUECOS
}

\author{
Salvador TALAVERA, Montserrat ARISTA, Pedro Luis ORTIZ y Fernando BASTIDA
}

\begin{abstract}
RESUMEN. Notas cariológicas sobre algunas compuestas de Marruecos. En esta nota se dan a conocer los números cromosómicos somáticos de 11 especies norteafricanas de la familia Compositae. Al parecer, es la primera vez que se estudia cariológicamente Atractylis humilis $\mathrm{L} .(2 \mathrm{n}=20)$ y de la mayoría de las especies es el primer recuento en material de Marruecos.
\end{abstract}

Palabras clave: Compositae, cariología, Marruecos.

SUMMARY. Kariological notes on some Compositae from Morocco. The chromosome number of eleven species of the family Compositae from Morocco are reported in this paper. It seems that Atractylis humilis L. $(2 n=20)$ has never been studied before from the cariological point of view. For most of the remaining taxa this is the first count in Morocco material.

Key words: Compositae, caryology, Morocco.

El estudio cariológico se ha realizado en meristemos radicales obtenidos de semillas recolectadas en Marruecos en otoño de 1993, cuyos testigos se encuentran depositados en el herbario del Departamento de Biología Vegetal y Ecología de la Universidad de Sevilla (SEV). Las raices se trataron con 8hidroxiquinoleína $0,002 \mathrm{M}$ a $4^{\circ} \mathrm{C}$ durante $3 \mathrm{~h}$ $30^{\prime}$ y se fijaron en alcohol etílico con ácido acético glacial (3:1) durante 24 h, realizándose la tinción siguiendo la técnica descrita por Snow (1963).

Aster squamatus (Sprengel) Hieron

$$
2 \mathrm{n}=20
$$

MARRUECOS: Al-Hoceima. Cerca de AlHoceima, margen del pantano del rio Nekor,
150 m.s.m., $35^{\circ} 6^{\prime} \mathrm{N}$ y $3^{\circ} 49^{\prime} \mathrm{W}, 2-\mathrm{XI}-1993, \mathrm{P}$. García Murillo, P. E. Gibbs \& S. Talavera, 197/93M (SEV 135443). El número somático encontrado en esta especie coincide con el hallado por numerosos autores (véase Pastor, 1992).

Atractylis humilis $\mathrm{L}$.

$2 \mathrm{n}=20$ (fig. 1,1 )

MARRUECOS: Taza, entre Aknoul y Tizi-Ouzli, 1000 m.s.m., $34^{\circ} 44^{\prime} \mathrm{N}$ y $3^{\circ} 51^{\prime} \mathrm{W}$, 2-XI-1993, P. García Murillo, P. E. Gibbs \& S. Talavera, 187/93M (SEV 135451). Al igual que otras especies del género Atractylis L., A. humilis L. tiene 20 cromosomas en sus células somáticas. Al parecer es la primera vez que se estudia la cariología en esta especie. 


\section{Carlina lanata $\mathrm{L}$.}

$2 \mathrm{n}=20$ (fig. 1,2 )

MARRUECOS: Taza. Entre Taza y AïnBou-Kellal, a $141 \mathrm{Km}$ de Al-Hoceima. Suelo yesoso, 500 m.s.m., $34^{\circ} 17^{\prime} \mathrm{N}$ y $3^{\circ} 58^{\prime} \mathrm{W}, 2-$ XI-1993, P. García Murillo, P. E. Gibbs \& S. Talavera, 134/93M (SEV 135449). El número cromosómico encontrado coincide con el hallado por Arata (1944) en plantas de Sicilia. Nuestro recuento, en material norte africano, confirma dicho número.

\section{Carlina racemosa $\mathrm{L}$.}

$2 n=20$ (fig. 1, 3)

MARRUECOS: Taza. Entre BabMarzouka y Taza, 400 m.s.m., $34^{\circ} 14^{\prime} \mathrm{N}$ y $4^{\circ} 7^{\prime}$ W, 31-X-1993, P. García Murillo, P. E. Gibbs \& S. Talavera , 61/93M (SEV 135450). El número cromosómico encontrado coincide con el hallado por Fernandes \& Queirós (1971) y Devesa (1979) en poblaciones de la Península Ibérica. Al parecer es el primer recuento hecho con material norteafricano.

\section{Centaurea diluta Aiton}

$$
2 n=20 \text {. }
$$

MARRUECOS: Chefchaouen. Entre Ketama y Bab-Berret, cedral, orilla de una torrentera, 2000 m.s.m., $34^{\circ} 58^{\prime} \mathrm{N}$ y $4^{\circ} 43^{\prime} \mathrm{W}$, 3-X-1993, P. García Murillo, P. E. Gibbs \& S. Talavera, 254/93M (SEV 135444). El número cromosómico encontrado en esta población rifeña es el mismo que el indicado por Blanca (1983) en plantas de Cádiz y Sevilla (bajo Centaurea micracantha). También es concordante con los números gaméticos, $\mathrm{n}=$ 10, indicados por Powel et al. (1973) y Ubera (1981).

\section{Cladanthus arabicus (L.) Cass.}

$$
2 n=18
$$

MARRUECOS: Taza, entre BabMarzouka y Taza, 400 m.s.m., $34^{\circ} 14^{\prime} \mathrm{N}$ y $4^{\circ} 7^{\prime}$ W, 23-X-1993, P. García Murillo, P. E. Gibbs \& S. Talavera , 65/93M (SEV 135447). El número somático encontrado se corresponde con el gamético hallado por Harling (1950) y por Talavera et al. (1984) en material de Marruecos.

\section{Dittrichia graveolens (L.) Greuter $2 n=18$}

MARRUECOS: Al-Hoceima. Cerca de AlHoceima, margen del pantano del rio Nekor, 150 m.s.m., $35^{\circ} 6^{\prime} \mathrm{N}$ y $3^{\circ} 49^{\prime} \mathrm{W}, 2-X I-1993, \mathrm{P}$. García Murillo, P. E. Gibbs \& S. Talavera, 199/93M (SEV 135442). El número somático encontrado en esta especie coincide con el observado por Fernandes \& Queirós (1971) en material de Portugal, Podlech \& Barder (1974) en plantas de Afganistán y Pavone et al. (1981) en material de Sicilia. Al parecer es la primera vez que se estudia esta especie en el norte de Africa.

\section{Hypochaeris radicata $\mathrm{L}$.}

$$
2 n=8
$$

MARRUECOS: Taza, entre Aknoul y Tizi-Ouzli, 1000-1400 m.s.m., 34 $44^{\circ} \mathrm{N}$ y $3^{\circ}$ 51' W, 2-XI-1993, P. García Murillo, P. E. Gibbs \& S. Talavera, 182/93M (SEV 135445). El número somático encontrado en esta especie coincide con los recuentos realizados por numerosos autores (véase Pastor, 1992) pero difiere del número somático $2 \mathrm{n}=28$ encontrado por Bir \& Santosh Kumari (1978) en material de Portugal.

Lactuca viminea (L.) F. W. Schmidt subsp. ramosissima (All.) Bonnier

$$
2 \mathrm{n}=18 \text { (fig. 1, 4) }
$$

MARRUECOS: Taza. Entre Aknoul y Tizi-Ouzli, 1000-1400 m.s.m., 34 44' N y $3^{\circ}$ 51' W, 2-XI-1993, P. García Murillo, P. E. Gibbs \& S. Talavera, 180/93M (SEV 135448). Los distintos autores que han estudiado las razas de Lactuca viminea han encontrado siempre $2 n=18$, número coincidente con el hallado por nosotros en la subsp. ramosissima. En la Península Ibérica ha sido estudiada por 

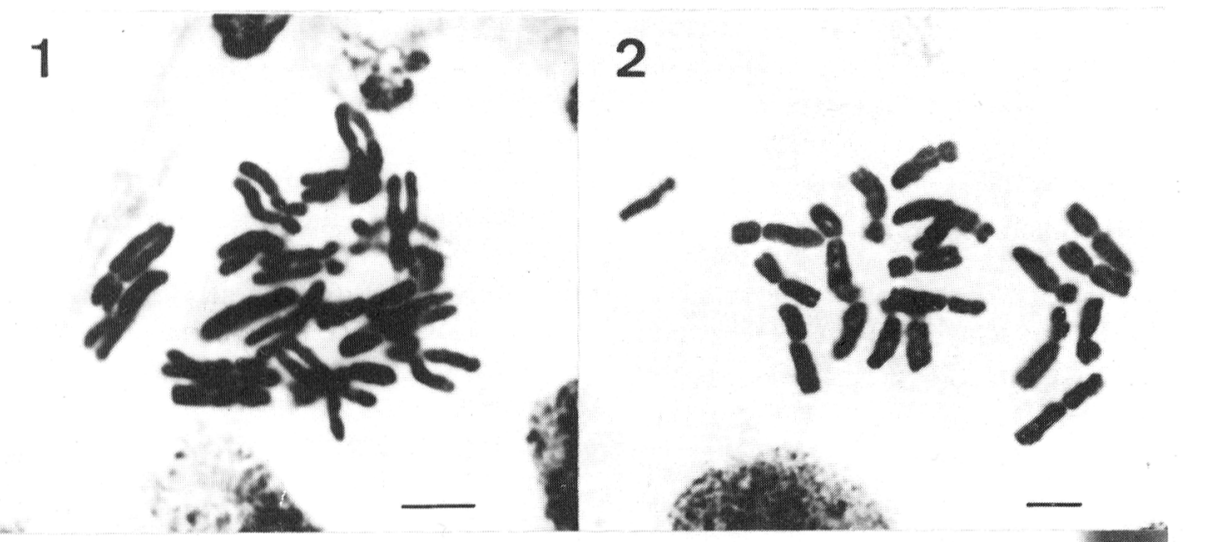

3

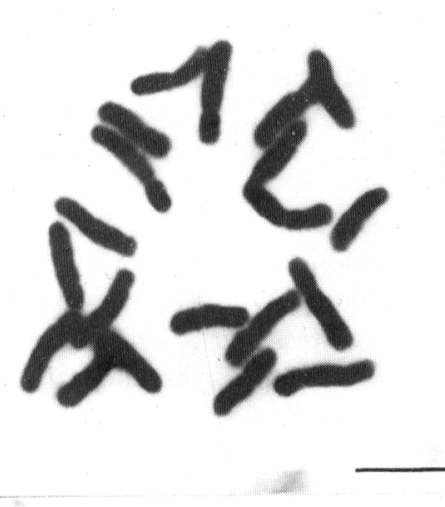

5
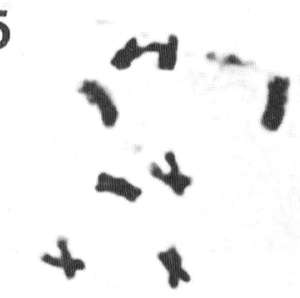

מ
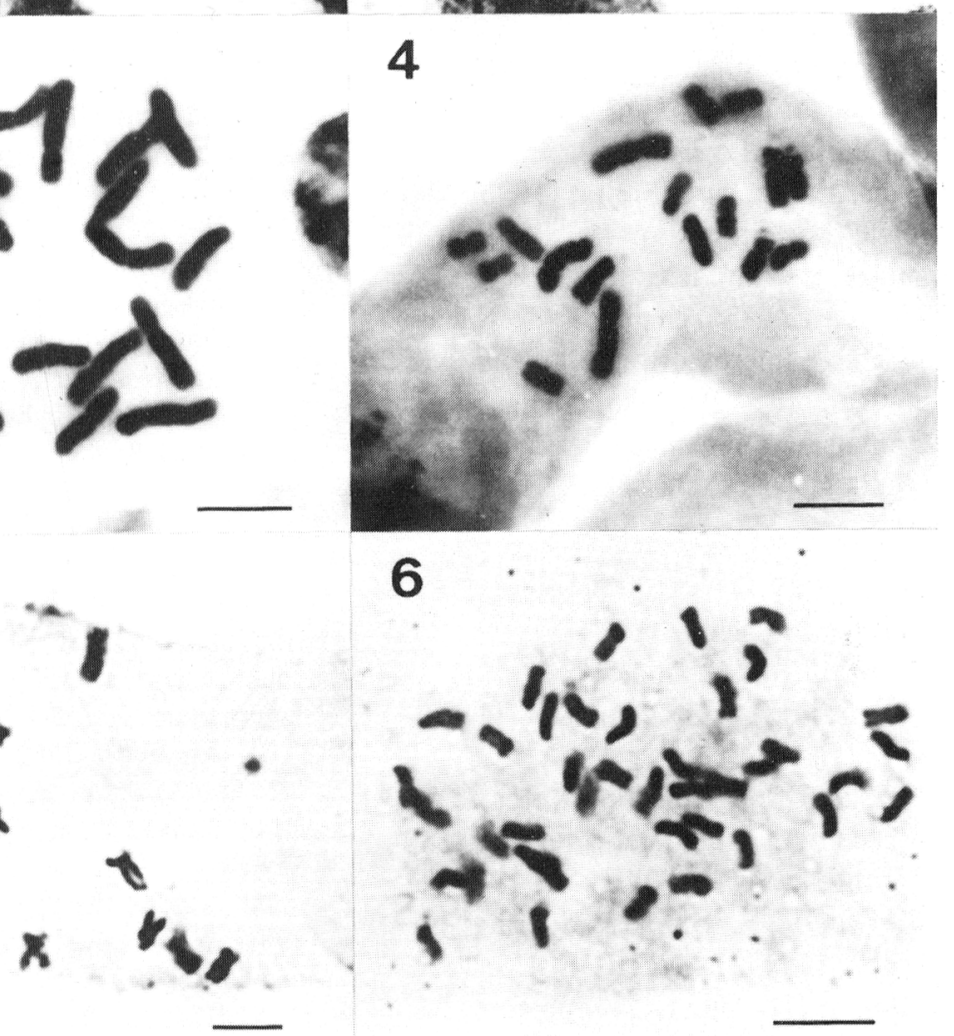

6

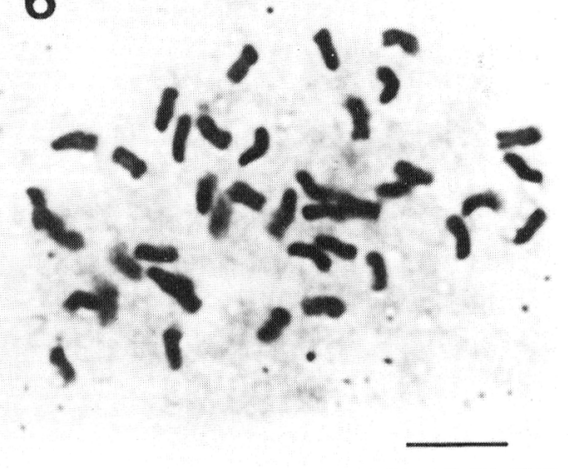

Figura 1. Metafase somática de (1) Atractylis humilis L., $2 \mathrm{n}=20$; (2) Carlina lanata L., $2 \mathrm{n}=20$; (3) Carlina racemosa L., $2 \mathrm{n}=20$; (4) Lactuca viminea (L.) F. W. Schmidt subsp. ramosissima (All.) Bonnier, $2 n=18$; (5) Launaea arborescens (Batt.) Murb., $2 n=14$ (5); (6) Senecio malacitanus Huter, $2 \mathrm{n}=40$ (SEV 135452). Escala: $5 \mu \mathrm{m}$. Somatic metaphase of (1) Atractylis humilis L., $2 n=20$; (2) Carlina lanata L., $2 n$ $=20$; (3) Carlina racemosa $L ., 2 n=20$; (4) Lactuca viminea (L.) F. W. Schmidt subsp. ramosissima (All.) Bonnier, $2 n=18$; (5) Launaea arborescens (Batt.) Murb., $2 n=14(5)$; (6) Senecio malacitanus Huter, $2 n=40$ (SEV 135452). Scale: $5 \mu \mathrm{m}$. 
Fernandes \& Queirós (1971), Queirós (1983), Silvestre (1984) y Mejías (1993a), no encontrando este último autor diferencias en los idiogramas de las tres subespecies analizadas. $\mathrm{Al}$ parecer es la primera vez que se estudia esta especie en poblaciones Norteafricanas.

Launaea arborescens (Batt.) Murb.

$$
\text { 2n }=14 \text { (fig. 1, 5) }
$$

MARRUECOS: Al-Hoceima. Entre TiziOuzli y Kassita, ramblas, 700 m.s.m., 34 $50^{\prime}$ N y $3^{\circ} 43^{\prime} \mathrm{W}, 2-X I-1993, \mathrm{P}$. García Murillo, P. E. Gibbs \& S. Talavera, 160/93M (SEV 135446). El número somático encontrado $2 n=$ 14 coincide con los hallados por Borgen (1969) en material de las Islas Canarias, Stebbins et al. (1953) de Argelia, y por Mejías (1993b) del SE de España (Murcia y Almería). También es concordante con el número gamético $n=7$, hallado por Reese (1957) en plantas del Sahara argelino. Estos números difieren del $2 \mathrm{n}=16$ hallado por Romero et al. (1985) en una población de Granada. Al parecer es la primera vez que se estudia la cariología de esta especie en Marruecos.

\section{Senecio malacitanus Huter}

$=S$. linifoliaster G. López

$=S$. linifolius Auctores non L.

$2 \mathrm{n}=40$ (fig. 1, 6)

MARRUECOS: Al-Hoceima. Cerca de AlHoceima. Cola del pantano del rio Nekor, 150 m.s.m., 35 6' N y $3^{\circ} 49^{\prime} \mathrm{W}, 2-\mathrm{XI}-1993$, P. García Murillo, P. E. Gibbs \& S. Talavera, 196/93M (SEV 135453). MARRUECOS: Taza. Entre Aknoul y Tizi-Ouzli, 1400 m.s.m., $34^{\circ}$ 44' N y $3^{\circ} 51^{\prime}$ W, 2-XI-1993, P. García Murillo, P. E. Gibbs \& S. Talavera, 175/93M (SEV 135452). El número cromosómico encontrado coincide con el hallado por Dahlgren et al. (1971) en material procedente de las Islas Baleares (bajo Senecio linifolius). Al parecer es la primera vez que se estudia esta especie en el norte de Africa.

\section{BIBLIOGRAFÍA}

ARATA, M. -1944- Sulla cariologia del genere Carlina L. Nouvo Giorn. Bot. Ital., 51: 39-43.

BIR, S. S. \& SANTOSH KUMARI -1978- In A. Löve (ed.), IOPB Chromosome number reports, LIX. Taxon, 27: 57-60.

BORGEN, L. -1969- Chromosome numbers of vascular plants from Canary Islands, with special reference to the occurrence of polyploidy. Nytt. Mag. Bot., 16: 81-121.

BLANCA, G. -1983- Números cromosomáticos de plantas occidentales, 234-238. Anales J. Bot. Madrid, 40: 257-261.

DAHLGREN, R., KARLSSON Th. \& LASSEN, P. -1971- Studies on the flora of the Balearic Islands. I. Bot. Not., 124: 249-269.

DEVESA, J. A. -1979- Números cromosómicos para la flora española, 110-115. Lagascalia, 9: 126-128.

FERNANDES, A. \& QUEIROS, M. -1971Contribution à la connaissance cy totaxinomique des Spermatophyta du Portugal. II. Compositae. Bol. Soc. Brot., ser 2, 45: 5-121.

HARLING, G. -1950- Embryological studies in the Compositae. Part. I. Anthemidae-Anthemidinae. Acta Hort. Berg., 15: 135-168.

MEJÍAS, J. A. -1993a- Cytotaxonomic studies in the Iberian taxa of the genus Lactuca (Compositae). Bot. Helv., 103: 113-130.

MEJIAS, J. A. -1993b- Estudio cariológico del género Launaea Cass. en la Península Ibérica. Lagascalia, 17: 135-149.

PASTOR, J. E. (ed.) -1992-Atlas cromosómico de la Flora Vascular de Andalucia Occidental. Publicaciones de la Universidad de Sevilla. Sevilla.

PAVONE, P., TERRASI, C. M. \& ZIZZA, A. 1981- In A. Löve (ed.), IOPB Chromosome number reports, LXXII. Taxon, 30: 695-696.

PODLECH, D. \& BARDER, O. -1974Chromosomenstudien an Afghanischen pflanzen II. Mitt. Bot. Staatssamml. München, 11: 457488.

POWEL, A. M., KYHOS, D. W. \& RAVEN, P. H. 1974- Chromosome numbers in Compositae. X. Amer. J. Bot., 61: 909-913.

QUEIRÓS, M. -1983- Números cromosómicos para a flora portuguesa, 64-85. Bol. Soc. Brot., Ser 2, 56: 79-89. 
REESE, G. -1957- Uber die Polyploidiespektren in der Nord saharischen Wüstenflora. Flora, 144: 598-634.

ROMERO, A. T., BLANCA, G. \& CUETO, M. 1985- Números cromosomáticos de plantas occidentales, 315-321. Anales Jard. Bot. Madrid, 42: 2 21-225.

SILVESTRE, S. -1984- Números cromosómicos para la flora española, 353-362, Lagascalia, 12: 298-303.

SNOW, R. -1963- Alcoholic hydrochloric acidcarmine as a stain for chromosomes in squash preparations. Stain Technol., 38: 9-13.

STEBBINS, G. L., JENKINS, J. A. \& WALTER, M. S. -1953- Chromosomes and phylogeny in the Compositae, Tribe Cichorieae. Univ. California Publ. Bot., 26: 401-430.
TALAVERA, S., DEVESA, J. A. \& FERNÁNDEZ GALIANO, E. -1984- Notas cariosistemáticas sobre plantas norteafricanas. I. Compositae. Candollea, 39: 271-280.

UBERA, J. L. -1981- Números cromosómicos para la flora española, 214-219. Lagascalia, 10: 237 239.

Aceptado para su publicación en Febrero de 1994

Dirección de los autores. S. Talavera, M. Arista y P.L. Ortiz: Departamento de Biología Vegetal y Ecología, Universidad de Sevilla, Apartado 1095. 41080 Sevilla. F. Bastida: Escuela Politécnica Superior de la Rábida. Departamento de Ciencias Agroforestales. Apartado 21710. Huelva. 